
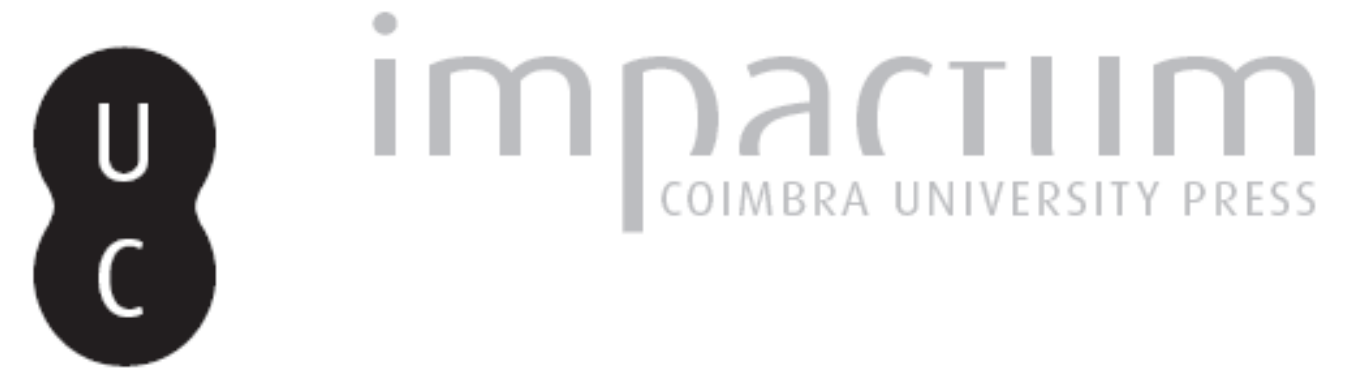

Translation revisited: ritorno alle traduzioni italiane della poesia di Pessoa

Autor(es): $\quad$ Mulinacci, Roberto

Publicado por: Imprensa da Universidade de Coimbra

URL persistente: URI:http://hdl.handle.net/10316.2/42501

DOI: $\quad$ DOI:https://doi.org/10.14195/0870-8584_7_8

Accessed : $\quad$ 26-Apr-2023 09:59:29

A navegação consulta e descarregamento dos títulos inseridos nas Bibliotecas Digitais UC Digitalis, UC Pombalina e UC Impactum, pressupõem a aceitação plena e sem reservas dos Termos e Condições de Uso destas Bibliotecas Digitais, disponíveis em https://digitalis.uc.pt/pt-pt/termos.

Conforme exposto nos referidos Termos e Condições de Uso, o descarregamento de títulos de acesso restrito requer uma licença válida de autorização devendo o utilizador aceder ao(s) documento(s) a partir de um endereço de IP da instituição detentora da supramencionada licença.

Ao utilizador é apenas permitido o descarregamento para uso pessoal, pelo que o emprego do(s) título(s) descarregado(s) para outro fim, designadamente comercial, carece de autorização do respetivo autor ou editor da obra.

Na medida em que todas as obras da UC Digitalis se encontram protegidas pelo Código do Direito de Autor e Direitos Conexos e demais legislação aplicável, toda a cópia, parcial ou total, deste documento, nos casos em que é legalmente admitida, deverá conter ou fazer-se acompanhar por este aviso. 


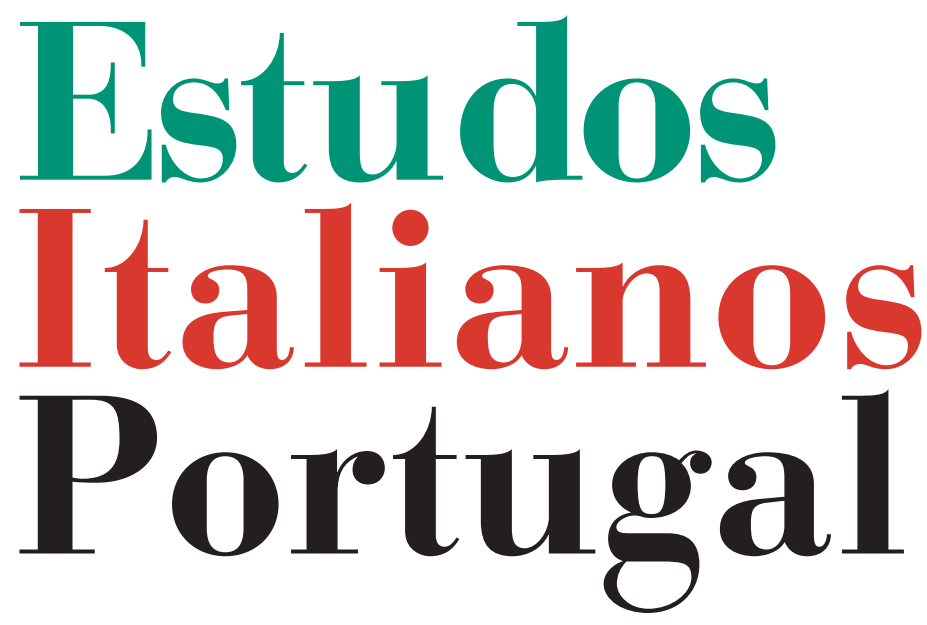

Instituto

Italiano

de Cultura

de Lisboa

Nova Série

$\mathbf{N}^{\mathbf{0}} 7$

2012 


\title{
TRANSLATION REVISITED. RITORNO ALLE TRADUZIONI ITALIANE DELLA POESIA DI PESSOA
}

\author{
Roberto MulinaCCI ${ }^{\star}$
}

\begin{abstract}
Outra vez te revejo Mas, ai, a mim não me revejo! Partiu-se o espelho mágico em que me revia idêntico, E em cada fragmento fatídico vejo só um bocado de mim Um bocado de ti e de mim!...

Lisbon Revisited, 1926
\end{abstract}

Quella che qui di seguito propongo è un'ideale continuazione di un "viaggio" cominciato esattamente venti anni fa. Era, infatti, il 1992 quando, nel primo dei tre volumi complessivi editi da Bulzoni nell'ambito di un progetto strategico del CNR sulla traduzione ${ }^{1}$, usciva, a firma di Gianluca Miraglia, un approfondito studio dal titolo "Le traduzioni italiane di Álvaro de Campos", che ripercorreva - traguardandola, in particolare, dall'ottica del suo "eteronimo più scandalistico" 2 - la storia della ricezione di Pessoa in Italia (o forse, sarebbe meglio dire, in italiano, visto che il primo atto di questa

\footnotetext{
* Professore associato di Lingua e Linguistica Portoghese e Brasiliana all'Università di Bologna

${ }^{1}$ Mi riferisco naturalmente al Progetto Strategico del Consiglio Nazionale delle Ricerche, all'epoca coordinato dal prof. Sergio Zoppi dell'Università di Torino e i cui risultati investigativi, suddivisi per aree linguistiche, erano appunto confluiti, almeno relativamente all'ambito iberistico (facente capo al prof. Giuseppe Bellini), nella pubblicazione di tre interessanti volumi dal titolo collettivo Del Tradurre, distinti solo da un numerale cardinale indicante la progressione della serie. Il contributo di Gianluca Miraglia qui citato ("Le traduzioni italiane di Álvaro de Campos") si trova, come detto, in Del Tradurre: 1, alle pagine 161-176.

${ }^{2}$ La definizione di Álvaro de Campos come "l'eteronimo più scandalistico di Pessoa" è di Luciana Stegagno Picchio (cfr. "Pessoa: uno e quattro", Nel segno di Orfeo. Fernando Pessoa e l'Avanguardia portoghese, Genova, Il Melangolo, 2004, p. 28).
} 
storia - come, del resto, altri dopo di esso $^{3}$-, si era comunque compiuto all'estero, sia pure in quella sorta di enclave extraterritoriale che sono da sempre gli Estudos Italianos em Portugal, sulle cui pagine, ben più di mezzo secolo addietro, Pessoa aveva fatto, appunto, il suo esordio nella nostra lingua, figurando in una piccola rassegna di poesia portoghese novecentesca, curata da Enzio di Poppa Vòlture). Una storia, quella ricostruita da Miraglia, invero assai selettiva, in quanto non si limitava al dato fattuale del testo tradotto - con il suo inserimento all'interno della realtà editoriale e, lato sen$s u$, culturale del Belpaese - né tanto meno si accontentava di ripiegare sull'accoglienza del poeta degli eteronimi presso gli addetti ai lavori della stampa specialistica e non, ma si concentrava, secondo copione, con competenza e puntiglio filologico, sulla qualità delle traduzioni praticate dai nostri pessoani della prima ora, ossia, da quell'avanguardia di inconsapevoli alfieri di un'incipiente pessoamania italica, alla cui definitiva esplosione, però, nel corso degli anni Ottanta, solo alcuni di loro avrebbero poi contribuito in modo concreto.

Penso, fra tutti, al principale artefice di questo inatteso e duraturo successo letterario, vale a dire, al compianto Antonio Tabucchi, la cui crestomazia pessoana (Una sola moltitudine, apparsa, per i tipi di Adelphi, tra il 1979 e il 1984, in 2 volumi, giunti oggi, rispettivamente, alla quattordicesima e dodicesima edizione) - che seguiva quella pionieristica di Luigi Panarese, pubblicata da Lerici nel 1967 e alla quale va riconosciuto, se non altro, il merito di aver fatto da catalizzatore $^{4}$, più che da semplice capostipite, alla nutrita schiera di

${ }^{3}$ Cfr. Luca Frediani, "La poesia di Fernando Pessoa" in Estudos Italianos em Portugal, 14-15, 1955-56, pp. 145- 168, e Orietta Del Bene, "L'Ode maritima di Fernando Pessoa”, Estudos Italianos em Portugal, 33-34-35, 1970- 71-72.

${ }^{4} \mathrm{Mi}$ riferisco, per esempio, alle circostanze - a cui accennava lo stesso Miraglia (cfr. cit., p. 170), che, hanno contribuito, almeno in minima parte, alla genesi dell'antologia di Giuseppe Tavani, Da Pessoa a Oliveira: la moderna poesia portoghese, Milano, 1973, nella quale l'ampia sezione di testi pessoani è, per esplicita ammissione 
analoghe iniziative antologiche - ha rappresentato un autentico punto di svolta nella ricezione di Pessoa in Italia, segnando, nella fattispecie, quello che Miguel Ángel Cuevas, con felice sintesi esegetica, ha definito il passaggio dalla fase "del mito a quella della conoscenza"s del grande poligrafo lusitano. E non è dunque un caso che proprio Una sola moltitudine costituisse l'emblematico approdo della ricognizione traduttologica di Miraglia, come a sancire - anche sotto questo peculiare aspetto analitico (e nonostante, direi, i puntuali rilievi dell'estensore dell'articolo, in generale condivisibili) - un significativo progresso nei confronti della maggioranza delle sillogi precedenti, con l'unica eccezione, forse, della miscellanea poetica curata da Tavani, le cui versioni pessoane sembrano, in effetti, contendere a quelle di Tabucchi il primato della qualità, soprattutto sul versante della loro tenuta ritmica in italiano ${ }^{6}$.

Certo, da allora, da quel 1992 assunto come terminus a quo della presente rassegna, molta acqua è passata sotto i ponti della critica pessoana e, anche in Italia, la fortuna del poeta plurimo ha potuto giovarsi dei nuovi orizzonti ermeneutici aperti da una sterminata bibliografia, che ha proceduto a larghe falcate al capillare dissodamento epistemologico dell'intero universo biografico-letterario del Nostro. Eppure, a dispetto della mole di pubblicazioni scientifiche e di edizioni divulgative made in Italy che hanno dato ulteriore linfa, in questo ultimo ventennio, al mainstream del pessoaismo internazionale (sebbene con apporti non sempre euristicamente entusiasmanti né editorialmente fondamentali, spesso ottenuti, anzi - parlando delle traduzioni -, per

del curatore, una sorta di ideale riparazione dei torti subiti da Pessoa nella summenzionata edizione di Panarese, la quale, sempre secondo Tavani, "non rende giustizia alla sua grandezza e alla sua originalità."

${ }^{5}$ Miguel Ángel Cuevas, "La Fortuna de Fernando Pessoa en Italia. del Mito al Conocimiento (1945-1979)”, Philologia Hispalensis, 4, 1989, pp. 707-721.

${ }^{6}$ Cfr. Miraglia, cit., pp. 170-172. 
gemmazione di repertori antologici già comparsi in italiano e successivamente autonomizzatisi, nel sottobosco del mercato librario nostrano, in singole plaquettes ritradotte ${ }^{7} \mathrm{o}$, magari, in repêchages, aumentati con materiali di riporto ${ }^{8}$ ), si contano davvero sulle dita di una mano gli interventi che si soffermano sui problemi traduttologici, a monte e a valle, concernenti le trasposizioni del corpus pessoano nella nostra lingua. Ad eccezione, infatti, di qualche sporadico accenno, racchiuso nei paratesti di raccolte poetiche recenti e sulle quali, dunque, tornerò meglio in seguito, le uniche riflessioni che io conosca - oltre, naturalmente, a quella da cui ha preso l'abbrivo questo mio scritto -, compiutamente dedicate al tema della traduzione di Pessoa in italiano, sono appena due?: il saggio di Luciana Stegagno Picchio, "Pessoa: uno e quattro" 10 , un piccolo classico, a suo modo, degli studi con-

${ }^{7}$ Cfr. F. Pessoa, Mare del Portogallo e altre poesie, trad. di Simonetta Masin e postfaz.di Martino Baldi, Pistoia, Edizioni Via del Vento, 2007, un libriccino di 35 pagine, in copie numerate, contenente nuove traduzioni, senza testo a fronte, di poesie tratte da Mensagem, nonché da Ricardo Reis, Alberto Caeiro e Álvaro de Campos.

${ }^{8}$ Sorvolando su certi casi di malcostume editoriale, probabilmente di responsabilità diretta degli editori, i quali tendono ad accreditare come "inediti" testi già usciti in traduzione italiana e a cui hanno aggiunto solo qualche frammento accessorio - è capitato qualcosa di simile con le novelas policiárias, tradotte dal sottoscritto nella loro originaria veste embrionaria e poi divenute oggetto di successive riedizioni in forma progressivamente accresciuta -, un repêchage classico della poesia pessoana è quello delle Poesie, a cura di Luigi Panarese, ma scelte dal "videopoeta" Giorgio Longo e pubblicate a Milano da Fabbri Editori nel 1997.

${ }^{9}$ A dire il vero, ci sarebbe anche il breve contributo di A. Tabucchi, "Traducendo "Il Marinaio", uscito dapprima a corredo della traduzione italiana omonima e poi ripubblicato in Un baule pieno di gente. Scritti su Fernando Pessoa (Milano, Feltrinelli, 1990, pp. 109-111) ma mi è parso che, per la sua natura autoriflessiva e, soprattutto, più strumentalmente funzionale, non fosse da includere in questo gruppo.

${ }^{10}$ Pubblicato, per la prima volta, in Strumenti Critici, 4, 1967, pp. 377-401. Ed è curioso, perciò, che Miraglia non l'abbia citato tra le fonti del suo testo, nonostante, ad onor del vero, le notazioni traduttologiche di Stegagno si circoscrivessero esclusivamente alla coeva antologia di Panarese (Milano, 1967), vale a dire, molto al di qua dell' ampio excursus diacronico che sarebbe stato poi tracciato, per l'appunto, in "Le traduzioni italiane di Álvaro de Campos". 
generi, con il quale, del resto, il lavoro di Miraglia condivide all'incirca le medesime preoccupazioni analitiche ${ }^{11}$ e quello di Giulia Lanciani, "Della traducibilità: il caso Pessoa"12, che di entrambi i suddetti potrebbe, invece, costituire una sorta di opportuna premessa metodologica. Se, tuttavia, sono costretto ad accantonare, in questa sede, per ovvi motivi ${ }^{13}$, la suggestiva tesi di Lanciani - che somiglia, a ben vedere, alla riproposizione, applicata al fenomeno Pessoa, della celebre "obiezione pregiudiziale", riguardante l' "interrogativo metafisico sulla possibilità della traduzione" 14 - e, nel contempo, a considerare la brillante lettura di Stegagno alla stregua di un'esperienza in sé conclusa, non tanto storicamente, quanto soprattutto testualmente, legata qual è ad un solo libro e ad un solo traduttore, è, viceversa, proprio la dimensione di potenziale work in progress implicita nell'articolo di Miraglia

\footnotetext{
${ }^{11}$ Ancorché, per esempio, le conclusioni sulla prassi traduttiva di Panarese - l'unico "minimo comune denominatore" tra Stegagno e Miraglia - non sempre convergano, però, perfettamente. In effetti, alle riserve, sia pure espresse con garbo, di Stegagno (la quale sottolineava una certa propensione del traduttore all'innalzamento di registro, a tratti quasi liricheggiante, rispetto alla cifra stilistica colloquiale dell'originale: cfr., in particolare, pp. 30-31), fa, invece, da parziale controcanto il laconico giudizio di Miraglia, secondo cui, "il tono prosaico, 'antilirico',viene reso adeguatamente” (p. 169).

${ }^{12}$ Uscito dapprima su Quaderno di Letterature Comparate, Università degli Studi di Roma Tre, 3, 2007, pp. 239-243, e poi ripubblicato, con una lieve, ma significativa variazione del titolo ("Dell'intraducibilità: il caso Pessoa") in Lingue Policentriche a confronto: quando il centro diventa periferia, a cura di G. L. De Rosa e A. De Laurentiis, Monza, Polimetrica International Scientific Publisher, 2009, pp. 13-19.

${ }^{13}$ In effetti, sebbene io concordi, complessivamente, con l'idea di una "poesia pessoana che si sottrae alla traducibilità" (p. 239) in modo quasi programmatico, cioè, per scelta consapevole dell'autore ("l'idiosincrasia di Pessoa nei confronti di ogni comprensione”, p. 240) oltre che per la sua peculiare vicenda editoriale, costellata di ricostruzioni postume che si sovrappongono aleatoriamente al precario dettato originario, resta il fatto che, dovendo qui trattare delle traduzioni italiane di Pessoa, non posso che partire dall'evidente, banale constatazione della loro esistenza, anziché dalla pur affascinante ipotesi contraria.

${ }^{14}$ Antonio Lavieri, Translatio in fabula. La letteratura come pratica teorica del tradurre, Roma, Editori Riuniti, 2007, p. 70.
} 
a renderlo suscettibile di nuovi sviluppi ed integrazioni. Ecco perché è da qui, come accennavo testé, che vorrei allora ripartire, dando, dunque, per acquisiti i risultati critici di chi mi ha preceduto e accontentandomi, tutt'al più, se ci riesco, di colmare quella lacuna, puramente cronologica, di cui il testo di Miraglia è indirettamente responsabile, avendo avuto il merito di indicare un percorso che forse vale ancora la pena di essere portato avanti.

Con un paio di precisazioni, però, sia di merito che di metodo. La prima ha a che fare con la qualità delle traduzioni pessoane ( $\mathrm{e}$, in fondo, delle traduzioni tout court), che, in Italia come altrove - complice, altresì, il significativo progresso dei Translation Studies -, è in questi anni migliorata molto, specialmente se la si confronta con quella della loro fase "preistorica", di cui si è occupato appunto Miraglia, affidata non di rado a specialisti di altre aree linguistico-culturali (i Gasparini, i Jannini, i Cicogna, i Negrelli, i Repetto ${ }^{15}$ ) e condizionata, più in generale (anche, cioè, a prescindere da polemici sciovinismi disciplinari), da un qualche difetto di consapevolezza relativo sia alle finalità della prassi traduttiva sia alle specificità del linguaggio poetico autoriale. Il che non significa, si badi bene, che un'analitica della traduzione di stampo, grosso modo, bermaniano, come quella praticata da Miraglia, sia diventata oggi inutile o perfino impossibile: assolutamente no. Solo che, nel profluvio di poesie ortonime ed eteronime, circolanti, con diversa fortuna, ai vari livelli dell'editoria italiana, dai piani nobili al sottoscala (absit iniuria verbis), la semplice collazione a campione delle traduzioni con gli originali - tesa ad individuare i luoghi testuali problematici di questo passaggio interlinguistico - comporterebbe

${ }^{15}$ Arrigo Repetto, a cui si deve la traduzione di alcuni componimenti di Caeiro, Reis e Campos inseriti nel suo Letteratura e Società nel Portogallo d'oggi (1865-1964) e un'antologia di autori portoghesi, Torino, ERI, 1965 - e poi ripubblicati in Antologia della letteratura portoghese, a cura di Cesco Vian, Milano, Fratelli Fabbri Editori, 1969 - è l'unico di questa serie a non comparire nella bibliografia del saggio di Miraglia. 
un dispendio di tempo e di energie forse non del tutto giustificato dai probabili esiti della rilevazione. Non che manchino - lo dico con cognizione di causa, a seguito di una lettura, per quanto cursoria, della stragrande maggioranza dei materiali in esame -, sviste ed errori $^{16}$ (taluni addirittura clamorosi) ${ }^{17}$ e, certo, alcune edizioni sono di gran lunga più affidabili di altre, ma, nel complesso, il grado di "fedeltà"18 del Pessoa italiano al suo verosimile omonimo portoghese (Lanciani docet) risulta abbastanza soddisfacente. Il resto sono dettagli, nel senso che, per quanto importanti ai fini della fruizione del testo pessoano, attengono, però, in massima parte

${ }^{16}$ Valgano come piccolo e bonario assaggio, coperti da rigoroso anonimato (ancorché evidentemente riconoscibili da parte dei vari traduttori e anche dai lettori più avveduti), i seguenti casi: "E asciugavano il sudore della testa accaldata", anziché della "fronte" (v. il port. testa dell'originale); "Gravi nomi vigilano i confini della consuetudini", dove l'originale (anch'esso riportato erroneamente) dice, invece, "Gravi numi" (port. numes); "Alla parca vita data anteponiamo/la novità, che è abisso" invece dell'esatto contrario (cfr.: "E a parca dada vida anteponhamos/à novidade, abismo"); "Credo nel mondo come a una pratolina, perché la vedo", mentre il clitico portoghese o, dello stesso genere di malmequer, si riferisce chiaramente - come si evince dal seguito - a mondo e non a pratolina. E l'elenco potrebbe continuare.

${ }^{17}$ In deroga ad un principio di diplomazia professionale che mi sono autoimposto, mi tocca purtroppo segnalare il fraintendimento davvero clamoroso dell'incipit di una poesia poco nota del Pessoa ortonimo, Flui, indeciso na bruma, che è stato tradotto con Sono fluito, indeciso nella bruma, scambiando, cioè, la terza persona singolare del presente indicativo di "fluir" (che si riferisce a "um ser que é coisa a achar/e a quem nada é preciso" dei vv.3- 4 e a cui rimanda poi l'intero componimento) con la prima persona del pretérito perfeito, ossia "fluî", reso, al modo consueto dell' italiano, mediante il passato prossimo. Ora, a parte la stranezza di quella nostra forma verbale, che, a quanto pare, dev'esser parsa, invece, alla traduttrice particolarmente consentanea a rappresentare lo stile eterodosso dell'autore, è soprattutto l' estraneità logica e sintattica di quel primo verso, che non c'entra nulla con il resto, a stridere pesantemente. E, poiché al peggio non c’è mai fine, la cosa paradossale è che questo errore marchiano è diventato il titolo della relativa plaquette! (Cfr. Fernando Pessoa, Sono fluito e altre poesie, Pistoia, Via del Vento, 2005).

${ }^{18}$ Uso questo termine, che personalmente aborrisco (almeno per quanto concerne l'ambito traduttologico), soltanto per comodità espositiva, essendo ben conscio della sua intrinseca scivolosità ermeneutica, che, appunto, in nome di un' illusoria evidenza semantica, riduce la complessità del tradurre alla logica elementare di una relazione biunivoca. 
al gusto personale del recensore e, dunque - al netto, altresì, dell'imbarazzo di chi scrive nel giudicare l'operato di colleghi e fatto salvo, a priori, il doveroso rispetto a cui ha comunque diritto l'attività traduttoria -, non meritano, credo, di figurare tra le pagine di queste brevi note, improntate, per converso, a criteri che vorrebbero essere di imparziale, quasi notarile oggettività (nella misura in cui, va da sé, una simile ambizione possa dirsi concretamente perseguibile).

Ne consegue, perciò, a proposito di metodo, che, lungi dal formulare giudizi sulle singole traduzioni o, peggio ancora, sui singoli traduttori (nomina sunt odiosa...), intendo, assai più modestamente, offrire uno sguardo d'insieme sul canone poetico pessoano che si è venuto affermando in Italia dal 1992 ai nostri giorni, grazie principalmente a quel combinato disposto costituito dalle case editrici e dalle università, alla cui feconda collaborazione si deve, in effetti, l'ampia scelta di opere, a volte di pregevole fattura, messa a disposizione di un crescente numero di ammiratori dello scrittore portoghese. D'altronde, è stato, perlopiù, tale classico connubio tra lo specialista e il "patronato"19, con i lusitanisti accademici nelle vesti di traduttori e editori, a garantire al grande pubblico, in questi anni, un Pessoa anche scientificamente valido, sottraendo così la celebre arca e i suoi numerosi tesori all'indiscriminato saccheggio della nostra industria culturale e svolgendo, di conseguenza, perfino una funzione di argine rispetto al dilettantismo di improvvisati cultori della materia ${ }^{20}$.

${ }^{19}$ Cfr. André Lefevere, Traduzione e riscrittura. La manipolazione della fama letteraria, Torino, UTET, 1998, p. 16: "Il secondo fattore di controllo, che agisce primariamente dall'esterno del sistema, verrà qui denominato 'patronato' intendendo con questo termine quei centri di potere (persone, istituzioni) in grado di favorire od ostacolare la produzione, la diffusione e la riscrittura di opere letterarie." Nel concetto di patronage lefeveriano rientra, dunque, a buon diritto anche la categoria degli editori.

${ }^{20}$ Senza voler fare una difesa corporativa della categoria (e, anzi, stigmatizzando qualunque forma di "protezionismo" editoriale, ancorché indirettamente esercitato), spiace, tuttavia, dover registrare che quanto più ci si allontana da questo ambito di specializzazione, tanto più probabile appare - soprattutto maneggiando un autore "dif- 
È, insomma, alla "fama letteraria" del Pessoa italiano e ai principali agenti della sua "manipolazione" 21 che guarda questo mio provvisorio bilancio, ben sapendo, però, che, a rigor di logica e in punta di lingua, non si tratta affatto di un ritorno - come io l'ho impropriamente definito nell'intestazione - bensì di una nuova partenza. Ma se diversi sono il movimento e, in ispecie, il tipo di sguardo, a volo d'uccello, con cui mi sono inserito nel solco di questo campo d'indagine, analoga è, per contro, la sensazione di spaesamento - stavolta, aggiungerei: di felice spaesamento - che, come in Lisbon Revisited, prende il non ignaro frequentatore di cose portoghesi nel rivedere cosa è diventato Pessoa oggi, anche in Italia: da "cittadella", appartata e quasi inaccessibile di un tempo non lontano, a "luogo" letterario di moda ed effettivamente "pieno di gente".

Sia chiaro: non rimpiango per nulla il Pessoa di nicchia, confinato nelle austere conventicole accademiche, appannaggio esclusivo di pochi eletti, né tantomeno il poeta sfingeo e un po' intellettualmente "autistico" dei suoi primi, sparuti interpreti italiani, al quale preferisco senza dubbio quello

ficile" quale Pessoa - quell'effetto "dilettanti allo sbaraglio" di certi prodotti commerciali di infimo ordine, usciti presso case editrici semisconosciute, la cui dimensione "minore" (di per sé, tutt'altro che negativa) non è, però, compensata da un adeguato pedigree culturale dei loro collaboratori. L'esempio emblematico, nella fattispecie, mi sembra possa essere rappresentato da Acquaviva, la cui sedicente edizione integrale del Pessoa ortonimo (L'esistenza spirituale. Tutte le poesie ortonime, Milano, 2009, un migliaio di pagine di traduzioni senza testo a fronte) è in realtà un florilegio di disinvolte parafrasi, piene di refusi ed amenità varie.

${ }^{21}$ Cfr. André Lefevere, cit., p. 10: "Traduzione, storiografia, antologizzazione, critica e revisione editoriale di testi letterari sono forme di riscrittura tutte regolate dal medesimo basilare processo, che è peraltro riscontrabile anche in altre forme di manipolazione testuale, come ad esempio, gli adattamenti per il cinema e la televisione, (...)." Tra le forme di "manipolazione" testuale indicate dallo studioso belga, il primato spetta senza dubbio alla "traduzione letteraria, che è la forma di riscrittura più evidente, oltre ad essere potenzialmente la più influente, in quanto capace di proiettare in un'altra cultura l'immagine di un autore e/o di una o più opere superando i confini della loro cultura d'origine." (ib.) 
dalle molte facce (oltre che dalle molte maschere) che una legione di traduttori ha contribuito a divulgare. Se, però, da lusitanista, il compiacimento è d'obbligo, considerato altresì che, all'inizio, Pessoa ha rappresentato l'autentico "cavallo di Troia" per la penetrazione della letteratura portoghese in Italia - salvo, purtroppo, arrivare poi quasi a sussumerla in $\operatorname{sé}^{22}$-, suggerisco comunque di non indugiare troppo sul rassicurante dato quantitativo arguibile dalla bibliografia qui di seguito in appendice e cercare piuttosto di comprenderne le dinamiche che gli sono sottese, evidenziando, cioè, un'ulteriore serie di dati (editori, curatori, collezioni, tiratura, vendite, testi d'accompagnamento), forse ben altrimenti indicativa della nostra reale ricezione pessoana, al di là del semplice numero di edizioni virtualmente presenti sul mercato. Va da sé, infatti, che talune traduzioni, apparse per i tipi di piccole case editrici, non possono, loro malgrado, vantare la stessa visibilità di quelle date alle stampe da marchi editoriali di livello nazionale e finiscono, quindi, inevitabilmente, per ridursi a mere posizioni bibliografiche, destinate ad un'esistenza semiclandestina fatta di tirature limitate e distribuzione precaria. Ora, posto che per certi titoli - secondo quanto ricordato sopra - la semiclandestinità non pare affatto una punizione eccessiva, ce ne sono altri, invece, per i quali essa risulta individualmente ingiusta e culturalmente svantaggiosa, non solo in ragione della buona qualità dei prodotti, ma altresì per il loro precipuo valore documentario, in grado di operare una salutare diffrazione dell'immagine del Pessoa maior imperante nella vulgata tradizionale, anche italiana. E' il caso, per esempio, dei due Pessoa "minori" curati da Sandro Naglia per le Edizioni Tracce di Pescara, ovvero, il poeta pseudopopolare delle Quadras, lì presentate per la prima volta in versione integrale, e il poeta inglese dell' Epitalamio, che, con un lustro

${ }^{22}$ Cfr. il mio "Come il Portogallo è diventato un' isola. La letteratura portoghese in traduzione italiana”, Estudos Italianos em Portugal, 6, 2011, pp. 179-185. 
di anticipo rispetto ai Trentacinque sonetti $i^{23}$, vi faceva il suo esordio in edizione separata $\mathrm{a}^{24}$. Tuttavia, più di questa doppia, felice circostanza editoriale e del progressivo allargamento d'orizzonte che essa ha comportato rispetto all'ineludibile centralità della galassia eteronimica, mette conto sottolineare, massime, gli anni della sua pubblicazione, rispettivamente il 1992 e il 1994, per me assurti, a posteriori, ad emblematica linea di displuvio della presenza pessoana in Italia, caratterizzata fino a quel momento da un sostanziale equilibrio, almeno numerico, tra le raccolte di poesia e i testi in prosa ${ }^{25}$. Si potrebbe, anzi, quasi dire, al riguardo, che, nonostante la fortuna di critica e di pubblico di Una sola moltitudine, il primitivo acclimatamento di Pessoa nel polisistema letterario italiano sia dunque avvenuto, paradossalmente, più sotto il segno dello scrittore "noir" che non del lirico metafisico, o, al limite, a voler essere precisi, sotto quello della loro contemporanea consustanziazione nel paradigma di una personalità

${ }^{23}$ F. Pessoa, I trentacinque sonetti, a cura di Ugo Serani, Firenze-Antella, Passigli Editori, 1999. Se si considera che nel 1989 era già uscito anche Il violinista pazzo (a cura di Amina di Munno, Roma, Lucarini, poi riedito da Mondadori nel 1995 e da Passigli nel 2004), appare dunque evidente che anche la poesia inglese di Fernando Pessoa è in Italia tutt'altro che negletta.

${ }^{24}$ L'altro elemento degli English Poems, Antinous, già tradotto da Kathleen Norris e Flavio Vaselli, era stato, infatti, inserito nel secondo volume di Una sola moltitudine (1984).

${ }^{25}$ Basta scorrere l'elenco delle traduzioni in volume per rendersene subito conto: in effetti, dal 1957, quando compare Il guardiano di greggi tradotto da Cicogna, al 1992, anno delle citate Quartine, si registrano sei antologie poetiche più il Faust e Il violinista pazzo, a fronte di quattro esemplari di narrativa "fantastica"(Due racconti del mistero, Il banchiere anarchico e altri racconti più due versioni distinte de L'ora del diavolo), due epistolari (Lettere alla fidanzata, Lettere a Ophélia), un dramma statico (Il Marinaio) e Il libro dell'inquietudine.

Se tuttavia si passa dalla "vertigine della lista" ad un suo tentativo, sia pure sommario, di interpretazione, si vedrà che, in realtà, c'è un analogo fil rouge che attraversa tutta questa produzione di genere vario e che rimanda più o meno al campo semantico dell'unheimlich freudiano, in cui si può forse condensare il senso di questa prima ricezione italiana di Pessoa. 
umana complessa, in cui la follia "razionalizzata e risolta"26 del discorso poetico sembrava trovare un prolungamento quasi fisiologico nelle atmosfere allucinate dei racconti del mistero ${ }^{27}$. Di fronte a queste due facce di una (praticamente) medesima medaglia, le quali, del resto, davano l'impressione di inverarsi proprio rispecchiandosi nell'apparente cortocircuito estetico tra l'anormalità patologica del letterato e la fittizia normalità dell'innamorato, si capisce meglio, allora, anche la simpatia con cui Tabucchi recensiva, sul Corriere della Sera del 27 dicembre 1992, l'uscita di quelle summenzionate Quartine di gusto popolare, che - ipse dixit - venivano ad aprire uno spiraglio di sana "genuinità" popolaresca sulla superficie dell'artificioso monumento poetico pessoano. Naturalmente, l'autore di Sostiene Pereira non si nascondeva di certo i risvolti altrettanto artificiosi di quel Pessoa "colto e intellettuale" che giocava a fare il "poeta amoroso", né, del resto, si peritava a cogliere il "senso dell'assurdo che colora questi versi popolareggianti di una tinta inquietante e bizzarra", rimarcandone, in fondo, l'implicita continuità tematica con l'opera maggiore. Cionondimeno, in quelle quartine portoghesi, assimilate, per l'occasione, ad autoctoni "stornelli", Tabucchi stava, in realtà, salutando - forse senza nemmeno saperlo - non tanto l'ennesimo travestimento del poeta plurimo da portare a conoscenza dei suoi lettori connazionali (c'era anche questo, ovvio), quanto, in particolare, quella sorta di autentico big bang della bibliografia italiana su Pessoa, che avrebbe cominciato esattamente da lì la propria inarrestabile e tumultuosa proliferazione.

Lungi dal voler ipotizzare indimostrabili rapporti di causaeffetto su fenomeni che sfuggono alle ambizioni di controllo

${ }^{26}$ A. Tabucchi, "Un baule pieno di gente", F. Pessoa, Una sola moltitudine, vol. I, 4. ${ }^{\text {a }}$ ed., Milano, Adelphi, 1991, p. 25.

${ }^{27}$ Cfr. L. Panarese, "Introduzione biografico-critica", F. Pessoa, Poesie, 4. ${ }^{\mathrm{a}}$ ed., Firenze, Passigli, 1996, p. 17: "l'equivalente verbale e figurato del proprio stato psichico riconosciuto nel genere letterario del mistero e dell'orrore". 
deterministico dei critici, resta comunque il fatto che il 1992 segna davvero l'anno della svolta nell'interesse dell'editoria nostrana per Pessoa, un interesse vieppiù diffuso e non episodico, come ci viene indirettamente confermato, oltre che dall'uscita delle Poesie di Álvaro de Campos - le quali, di questa svolta, sono il primo frutto maturo ${ }^{28}$-, dalla storia, per l'appunto, delle stesse Quartine, a cui, tra i testi poetici pessoani, spetta pure l'inatteso primato del numero di edizioni (ben quattro in appena un ventennio, con tre editori diversi e addirittura una doppia lezione testuale a fare da base per la traduzione ${ }^{29}$. Un dato, questo, forse di per sé irrilevante, ma che può diventare, magari, significativo, qualora lo si proietti fuori dallo specifico recinto filologico-commerciale in cui si inquadra, a testimoniare piuttosto il progressivo consolidarsi dell' appeal editoriale di Pessoa, reso ormai sempre più indipendente non solo dagli eventuali effetti di traino del suo mito (o di quel che ne resta), ma, purtroppo, anche dall'intrinseca realtà del fatto letterario che lo riguarda. In questo senso, credo che la superfetazione di quadras a disposizione dei pessoani doc d'Italia - al di là del giudizio di merito che se ne voglia dare (sulle quadras, chiaro, non sui loro ipotetici cultori) - valga innanzitutto come prova

${ }^{28}$ Primo non solo in linea genealogica tra gli eteronimi pubblicati individualmente, ma anche per la centralità che Álvaro de Campos dimostra di avere nella iniziale mappatura pessoana delle nostre antologie. Si pensi, come detto, al rilievo assegnatogli sia da quella di Tavani sia da quella di Tabucchi, il quale, tra l'altro, ne aveva fatto pure l'eponimo principale di un altro suo florilegio, Nove poesie di Álvaro de Campos e sette poesie ortonime, Bologna, Baskerville, 1988. Il volume Poesie di Álvaro de Campos, a cura di Maria José de Lancastre (e con traduzioni di Tabucchi), edito da Adelphi nel 1993 viene, dunque, a chiudere questa fase di rapsodica esplorazione della poesia di Pessoa, inaugurando ufficialmente quella della sua sistematica pubblicazione.

${ }^{29} \mathrm{Mi}$ riferisco, infatti, oltre all'edizione di Naglia (riveduta e ripubblicata nel 1999 per la collana "Voci in viaggio" del quotidiano L'Unità e poi, ulteriormente aggiornata nel 2011), a quella curata nel 2005 da Luciana Stegagno Picchio per Passigli, divenuto ormai l'editore italiano per antonomasia di Fernando Pessoa, di cui, da anni, sta traducendo con una certa regolarità tutte le opere pubblicate presso il suo omologo 
provata della forza autopropulsiva del "marchio" Pessoa, trasformato in griffe alla moda capace di imporre perfino le sue ipostasi letterarie consapevolmente - o meglio: "volutamente" (l'avverbio è di Tabucchi) - minori.

Lo ripeto, a scanso di equivoci: è un bene che ci siano diverse edizioni delle varie opere, perché è un indice dell'attenzione suscitata da una cultura o da un autore e anche perché, a volte, la pluralità di esemplari assolve ad un essenziale compito di reciproca integrazione. $\mathrm{Ma}$, insieme agli aspetti astrattamente positivi di questa non effimera voga pessoana del mercato librario italiano, non è male far cenno altresì ai loro concreti corrispettivi di segno opposto, i quali rientrano, perlopiù, in quello che chiamerei l'indotto editoriale legato al poeta degli eteronimi, ovvero, gli elementi materialmente coinvolti nella filiera produttiva dei suoi volumi, cioè, in primis, traduttori e curatori. Il moltiplicarsi dei titoli pessoani messi in circolazione dalla fine del secolo scorso in Italia ha, infatti, richiesto un sia pure saltuario rimpinguamento dei membri di quelle due categorie professionali, al quale, però, non ha sempre corrisposto un'adeguata preparazione lusitanistica dei medesimi, col risultato, dunque, di far perdere ai testi, in

lusitano, Assírio \& Alvim. Ebbene, le Quartine (così, semplicemente, senza appendici definitorie a delimitarne il registro stilistico) tradotte dalla Stegagno Picchio si basano sull'ultima edizione delle Quadras condotta a termine, sempre per Assírio e Alvim, da Luísa Freire e presentano, dunque, sostanziali differenze con quelle di Naglia - che, invece, prendono a riferimento la princeps di Georg Rudolf Lind e Jacinto do Prado Coelho -, a cominciare dal numero (due serie di componimenti, indicati come Quartine I e II, rispettivamente di 164 e 248 testi contro i 325 dell'edizione pescarese) e dai criteri editoriali, per finire - last but not least - con la strategia traduttiva che dà loro concretamente forma. In effetti, mentre Naglia e Mariavittoria Altieri si attengono a un principio di traduzione grosso modo "strumentale", teso soprattutto al mantenimento del senso, Stegagno Picchio, al contrario, opta per una traduzione più rispettosa altresì delle esigenze rimiche e ritmiche dell'originale, con esiti spesso eccellenti (tra l'altro, sulle difficoltà di traduzione delle Quadras, nonché sugli "artifici” usati per superarle, cfr. la bella Prefazione al volume, a firma della stessa Stegagno, nella fattispecie le pp. 12-14). 
precisione (traduttiva o informativa), quello che essi suppostamente guadagnavano in diffusione. E questo lo si vede, in modo particolare, nel settore delle pubblicazioni di poesia, penalizzato più di altri - per caratteristiche congenite al tipo di scrittura nonché alla forma mentis che presiede alla sua riformulazione idiolettale - da deficit di specifica competenza pessoana, a cui si è cercato di ovviare, qua e là, rifugiandosi in piatte traduzioni letterali ${ }^{30}$ (condite, al massimo, da qualche variatio sinonimica, giusto per sfuggire al calco pedissequo dei predecessori) e/o in trite compilazioni di luoghi comuni critici, le une e le altre giustificate, sì, dal prioritario intento divulgativo, ma probabilmente non in grado di giustificare del tutto anche il significato del loro stesso esistere. Senza contare, per giunta, che dal fenomeno, nella sostanza non deteriore, della ritraduzione - ritenuto, in genere, perfino necessario ai fini di un'attualizzazione del linguaggio traducente, benché, nella fattispecie, di fronte a intervalli di pochi anni tra una traduzione e l'altra, esso appaia evidentemente superfluo -, discende, per filiazione diretta, l'abusata pratica della riedizione $e^{31}$, spesso imposta dalle case editrici a puro scopo di lucro e consistente nel semplice riassemblaggio, sotto mentite spoglie, di materiali poetici già noti da raccolte anteriori. Se tale pessima usanza non è di sicuro un'esclusiva dell'industria culturale nostrana, dispiace, però, che a farne le

\footnotetext{
${ }^{30} \mathrm{Al}$ polo opposto del fenomeno si colloca, invece, quello delle traduzioni metriche, in cui la pur apprezzabile tendenza a rendere gli aspetti formali costitutivi del verso, quali il metro o il ritmo, ha talora finito per prendere la mano ad alcuni traduttori, che, sull'altare di quella fedeltà, hanno di quando in quando sacrificato il senso: cfr. "Lapso curto dos dias e se volve/à antiga liberdade/que talvez nunca houvemos" che diventa, in italiano, "Arco breve dei giorni per tornare/all'antica libertà/mai forse non avuta”, dove quell'avverbio negativo, usato da zeppa metrica- e a dispetto della ricercata licenza poetica -, inverte il significato dell'originale portoghese.

${ }^{31} \mathrm{Ma}$ il contrario non è sempre vero, nel senso che la riedizione non implica automaticamente la ritraduzione, come dimostra il volumetto delle Odi di Ricardo Reis, curato da Libero Corsi (Milano, La Vita Felice, 2007), il quale si limita a riprodurre - perfino nella stessa sequenza - le 36 Odi già pubblicate nell'antologia di Panarese.
} 
spese sia stato financo il corpus poetico del Grande Portoghese, smembrato e parcellizzato in una serie di libri e libriccini a tema, pensata appositamente per assecondare i gusti di un pubblico meno soggetto al fascino del nome Pessoa e più propenso, invece, a rispondere al richiamo di titoli di facile presa. Ecco, allora, che, dopo il Messaggio incluso nella solita antologia tabucchiana e ben prima della sua edizione individuale, realizzata dal solito Paolo Collo per Passigli ${ }^{32}$, Guanda dà alle stampe, nel 2000, per le cure del filologo romanzo Francesco Zambon - a cui si deve, oltre alla traduzione in collaborazione con Ivette Marli Boso, anche la densa introduzione ("Fernando Pessoa e l'oltre-Dio") -, il volume Poesie esoteriche, che riunisce, in una nuova struttura e con ulteriori innesti (editi e non), gran parte dei componimenti tratti proprio dal poema mistico-patriottico di cui sopra. La stessa cosa, del resto, farà anche Passigli qualche anno più tardi, riproponendo - in una nuova traduzione e, soprattutto, con un ammiccante e un po' dozzinale maquillage del titolo (Poemetti erotici) ${ }^{33}$-, sia i due poemetti "indecenti" già pubblicati da Adelphi e da Tracce (Antinoo e Epitalamio), sia una selezione di poesie di Ricardo Reis, tratte stavolta da una precedente edizione della casa, ma sottoposte ad identico processo di strategica rinominazione (Poesie d'amore di Ricardo Reis, a cura di Paolo Collo, 2007).

A proposito, tra l'altro, di quest'ultimo eteronimo - il quale, con Alberto Caeiro, guida, ex aequo (3 a 3) ${ }^{34}$, la spe-

${ }^{32}$ In realtà, Paolo Collo, autore dello scritto introduttivo ("I percorsi di Pessoa"), è il responsabile dell'edizione italiana di Mensagem curata per Assírio \& Alvim da Fernando Cabral Martins. A quest'ultimo, dunque, si deve, oltre alla postfazione, dal titolo "Noi, il Portogallo", anche l'organizzazione complessiva del testo tradotto. (Messaggio, Bagno a Ripoli, Passigli Editori, 2003).

${ }^{33}$ Poemetti Erotici, trad. di Marcello Cavallini, Bagno a Ripoli, Passigli, 2007. La pruderie piccolo-borghese della definizione riservata ai due poemi inglesi ("indecenti") è di Pessoa, che così li definiva, appunto, in una lettera del 1916 all'amico Côrtes-Rodrigues.

${ }^{34}$ A dire il vero sarebbero quattro a testa, ma ho espunto dal computo sia la prima edizione italiana in volume di Pessoa, Il guardiano di greggi, a cura di Enrico Cicogna, pubblicato dalle Officine Grafiche Esperia di Milano nel 1957 e oggi assolutamente 
ciale classifica del numero di volumi che gli sono singolarmente dedicati -, mi sembra che il trattamento riservatogli da Passigli si presti a qualche riflessione supplementare, dal momento che, in controtendenza rispetto alla comune prassi dell'editore, le Poesie di Ricardo Reis (uscite nel 2005 a cura di Manuela Parreira da Silva, con traduzioni di Laura Naldini e Virginiaclara Caporali ad affiancare quelle storiche dell'antologia di Panarese) risultano prive di qualunque apparato introduttivo, eccetto la scarna Nota finale, in cui la curatrice spiega sinteticamente i criteri dell'edizione (e le traduttrici, ancor più sinteticamente, in due sole righe!, quelli della traduzione). Ma se è già abbastanza singolare che l' "editio princeps" del poeta oraziano, in quanto porta d'ingresso principale a questa maschera e al suo canzoniere, non preveda la benché minima "guida di lettura", mentre il successivo campionario amoroso, che ne è un estratto, ne abbia una, ancor più singolare, forse, apparirà il fatto che, alla fin fine, sia la prefazione di Paolo Collo - lo dico con il massimo rispetto per l'eclettico traduttore di cose iberistiche - l'unico viatico ad usum del lettore italiano desideroso di addentrarsi nell'universo poetico di un gigante come Ricardo Reis. Anche perché proprio Passigli ci ha abituato, in questi anni, a libri pessoani di un certo pregio, con curatele affidate addirittura a rinomati specialisti internazionali, tra cui Fernando Cabral Martins ${ }^{35}$ e Richard Zenith, i quali, per esempio, hanno firmato in congiunto la bella edizione del-

irreperibile, sia, per questioni di genere letterario, le Prose di Ricardo Reis (Passigli, 2005), la cui presenza non si confa ad un articolo incentrato sui testi poetici. Comunque, per completezza informativa, bisogna aggiungere che de $O$ guardador de rebanhos è uscita nel 2007, ça va sans dire, da Passigli, una nuova edizione, a cura sempre di P. Collo e con traduzioni di Pierluigi Raule e Panarese.

${ }^{35}$ Oltre al succitato Messaggio, la collaborazione di Cabral Martins con Passigli sempre via Assírio \& Alvim - ha partorito anche un altro testo poetico interessante quale Fantasie di interludio, uscito nel 2001 e che costituisce il primo libro di poesia pessoana (in portoghese) pubblicato dall'editore, dopo i Trentacinque sonetti. 
le Poesie di Alberto Caeiro ${ }^{36}$, non limitandosi a ritrascrivervi testi e organizzarne le varianti, ma arricchendola perfino di due corposi e documentati saggi conclusivi ("Caeiro trionfale" di R. Z. e "La nozione delle cose" di F.C.M.). Da questo punto di vista, dunque, sebbene i vincoli derivanti a Passigli dalla partnership editoriale con Assírio \& Alvim - titolare, dal 1997 al 2005, dei diritti d'autore sull'opera di Pessoa possano spiegare meglio di tante affermazioni congetturali il confezionamento dell'edizione portoghese da parte della sua "licenziataria" italiana (imputando, perciò, alla fonte eventuali assenze ed omissioni), continuo, tuttavia, a ritenere che proprio una diversa valutazione della collocazione di Pessoa nel nostro contesto culturale - cioè, inevitabilmente meno radicata in confronto al Portogallo - avrebbe dovuto sconsigliare una lacuna critica come quella su Ricardo Reis.

A dispetto, però, di questi difetti di ricezione che ho sommariamente tratteggiato, bisogna, al postutto, riconoscere che il panorama odierno delle traduzioni di poesia pessoana in Italia è senza dubbio confortante. Il grosso della produzione ortonima ed eteronima vi si trova, infatti, doviziosamente rappresentato, in un'ampia gamma di proposte editoriali a prezzi accessibili, per tutte le tasche e per (quasi) tutti i gusti, dallo specialista al neofita, che potranno, dunque, apprezzare (o, magari, disapprovare) il diverso grado di accuratezza delle varie edizioni in misura direttamente proporzionale alle loro specifiche conoscenze della materia. Se si considera, poi, che il catalogo pessoano più significativo è ormai saldamente nelle mani di pochi, grandi editori - con buon profitto della reperibilità dei testi e a garanzia altresì della loro qualità - e che ultimamente i versi del Genio portoghese hanno preso a circolare persino al di fuori dei consueti canali di distribu-

${ }^{36}$ Le traduzioni di questa edizione sono di Pierluigi Raule, Ugo Serani e Laura Naldini. Tra l'altro, Raule aveva già pubblicato i Poemi di Alberto Caeiro nel 1999, per i tipi de La Vita Felice, di Milano (cfr. anche la recensione di Mario Andrea Rigoni sul Corriere della Sera del 23.07.1999: "Pessoa, ovvero il poeta A. Caeiro"). 
zione libraria, finendo addirittura nella prestigiosa collana di poesia del Corriere della Sera ${ }^{37}$, si potrà capire, allora, questo mio insolito indulgere all'ottimismo, soprattutto quando si tratta di delineare le sorti delle letterature lusofone in Italia. Certo, Pessoa, al pari di Saramago, è un'isola felice, in un mare nostrum abbastanza avaro di soddisfazioni per la cultura lusitana (chissà se sarebbe piaciuta, al "creatore di miti", questa banale metafora equorea...) e, in quanto tale, non fa letteralmente testo. Non lo fa nel senso che, nel subisso di traduzioni pessoane, manca probabilmente un testo capace davvero di identificare Pessoa per il lettore italiano, il quale, in questi anni, deve piuttosto essersi formato di lui un' immagine giustamente poliedrica, di funambolico virtuoso della scrittura, non meno autentico, quindi, nei panni del giallista che in quelli del poeta. Del resto, era un po' questa, mutatis mutandis, l'immagine che emergeva di Pessoa - ancorché con apporti documentali fatalmente più circoscritti al progetto poetico -, da quel preliminare sondaggio della sua opera presentato in Una sola moltitudine, forse ancora oggi l'unico, vero, grande "classico" della nostra biblioteca pessoana, a cui, peraltro, sembrano rimandare, nella loro impostazione complessiva, anche talune recentissime antologizzazioni, come, ad esempio, i due ponderosi volumi curati da Piero Ceccucci per la BuR ${ }^{38}$.

${ }^{37}$ Come quarto volume della collana "Un secolo di poesia" - che annovera altre voci prestigiose della poesia internazionale (dalla Szymborska a Kavafis, da Neruda a Borges, da Walcott a Ungaretti) - è, infatti, uscito nel 2011 Nei giorni di luce perfetta, a cura di Paolo Collo, contenente un'antologia di versi pessoani tratti dal succitato Fantasie di interludio, (v. nota n. 35). L' introduzione del volume, senza infamia e senza lode, era, però, dello scrittore Marco Missiroli. Questa edizione pessoana del Corriere della Sera segue, tra l'altro, quella del 2004 (come supplemento al giornale del 17-05-2004), Poesie, a cura di Panarese, ma con la prefazione di Marzio Breda, poi ripubblicata da Passigli nel 2006 in edizione rilegata (Poesie scelte).

${ }^{38}$ Il mondo che non vedo. Poesie ortonime, (2009) e Un'affollata solitudine. Poesie eteronime (2012). Del primo volume, impreziosito dalla postfazione di José Saramago, esiste anche una versione, per così dire, ridotta (152 pagine contro 999), dal semplice 
Effettivamente, dopo la comprensibile stagione antologica seguita alla scoperta italiana di Pessoa e il successivo passaggio a quella delle edizioni separate di ciascun eteronimo (almeno dei tre maggiori) e dell'ortonimo - stagione, questa, che va all'incirca dal 1992 al 2009 -, Il mondo che non vedo e Un'affollata solitudine segnano il ritorno ad un'idea di antologia che partecipa, a tratti scopertamente ${ }^{39}$, delle esperienze di quella prima fase, riuscendo, però, a riadattarle in modo efficace alle mutate esigenze ed aspettative del nostro attuale milieu culturale. Dietro, infatti, al tradizionale impianto di selezione e accorpamento dei testi, quantunque ricalibrato parzialmente nella rigida divisione dei versi ortonimi dagli eteronimi, la doppia edizione pessoana di Ceccucci offre non solo un bell' esempio di buona divulgazione scientifica - sulla scia, appunto, dei suoi illustri modelli -, ma anche di ben più esplicite ambizioni critiche, legittimate, io credo, principalmente da un'informazione di base su Pessoa che in Italia è già stata saturata e che, perciò, induce i suoi esegeti attuali a ulteriori sforzi di approfondimento. Ne fanno fede - di tali ambizioni critiche, intendo -, oltre agli apparati di note ("biobibliografica" 40 e "di edizione", quest'ultima particolarmente utile per dar conto sia dell'opportuna scelta ortografica arcaicizzante del testo a fronte, sia dello stile filologicamente conservativo della traduzione), i due puntuali saggi

titolo di Poesie, curato sempre da Ceccucci per la BUR, la quale l'ha pubblicato, in quello stesso 2009, in una mini-collana creata apposta per celebrare il sessantesimo anniversario della sua fondazione. Tra l'altro, in questa versione light, lo scritto di Saramago figurava come prefazione.

${ }^{39}$ Basti pensare all'omaggio citazionistico del titolo del secondo volume, che è una patente inversione a chiasmo del titolo dell'antologia tabucchiana, a sua volta citazione di Montale.

${ }^{40}$ La precisa Nota Biobibliografica, contenuta in Il mondo che non vedo, è di responsabilità di Orietta Abbati, la quale firma anche la seconda introduzione di Un'affollata solitudine, "Una vita declinata al plurale. Cenni sull'eteronimia pessoana", pp. XLIIILXXIX. 
introduttivi ${ }^{41}$ del curatore, che si potrebbero tranquillamente immaginare pubblicati su riviste specialistiche, nonostante la concessione al grande pubblico delle citazioni in italiano e la, purtroppo, inopinata mancanza di una bibliografia generale di riferimento. Ma sono soprattutto i 955 testi (di cui 618, in rigoroso ordine cronologico, solo dell'ortonimo), riprodotti in maggioranza dall'edizione critica dell'Imprensa Nacional, a costituire il valore aggiunto dei due volumi, che racchiudono, dunque, al momento, il più completo e affidabile repertorio di poesia pessoana disponibile sugli scaffali delle nostre librerie, così da poterne prefigurare, nell'immediato futuro, un utilizzo anche come valido strumento di supporto alla didattica universitaria.

E allora, giunti a questo punto, il mio viaggio può finalmente terminare. Siamo partiti per cercare di capire che volto avesse, in Italia, il Pessoa poeta e ci siamo imbattuti in una ridda di volti e di maschere che sembra fatta apposta per impedirci di scorgerne un'immagine prevalente - ammesso che esista -, proprio come per il "vero" Pessoa portoghese. In quest'ottica, dunque, direi che l'editoria italiana ha colto perfettamente nel segno, pluralizzando la ricezione di un autore che può vivere soltanto nell'entropia del suo sistema letterario, in un presente frammentario "determinato - per usare le parole di Benjamin - da quelle immagini che gli sono sincrone", in cui ogni ora "è l'ora di una determinata conoscibilità" ${ }^{2}$. A questo nostro presente pessoano, "la pellicola della traduzione" ${ }^{\text {"43 }}$ che vi si è

${ }^{41}$ Cfr. "Occhi per sentire... ragione per vedere". La conciliazione negata di sogno e raziocinio nella poesia ortonima di Fernando Pessoa" (in Il mondo che non vedo, pp. III-XXXVIII) e "'Ogni angolo dell'anima mia'. Pluridiscorsività e intratestualità nel discorso poetico eteronimo di Fernando Pessoa" (in Un'affollata solitudine, pp. III-XLI).

${ }^{42}$ W. Benjamin, Parigi capitale del XIX secolo. I passages di Parigi, Torino, Einaudi, 1986, p. 599.

${ }^{43}$ A. Tabucchi, "Traducendo 'Il Marinaio", Un baule pieno di gente. Scritti su Fernando Pessoa, p. 111. 
posata sopra non ha aggiunto, per fortuna, troppa, ulteriore opacità. Certo, non tutto è rose e fiori e, in fondo, pessoanamente, manca sempre qualcosa, ma - pur senza attingere a notizie aggiornate riguardanti la bibliografia delle traduzioni di altri paesi - ho la presunzione di affermare che in Italia si sia già fatto abbastanza per divulgare decentemente l'opera del poeta plurimo. Il che, lungi dall'essere un invito a tirare i remi in barca, vorrebbe piuttosto servire di sprone a nuove e ancora più accurate iniziative editoriali, capaci, magari, di ricomporre, frammento dopo frammento, quel mosaico chiamato Fernando Pessoa. Outra vez te revejo, ... 


\section{BIBLIOGRAFIA DELLE TRADUZIONI ITALIANE DI POESIA PESSOANA PUBBLICATE IN VOLUME}

Il guardiano di greggi, a cura di E. Cicogna, Milano, Officine Grafiche Esperia, 1957;

Poesie, a cura di L. Panarese, Milano, Lerici, 1967 (riedizioni: Firenze, Passigli Editori, 1993; scelta di Giorgo Longo, Milano, Fabbri Editori, 1997; con pref. di Marzio Breda, Milano, RCS-Corriere della Sera, 2004; Poesie scelte, Bagno a Ripoli, Passigli Editori, 2006);

Imminenza dell'ignoto, a cura di L. Panarese, Milano, Accademia, 1972;

Una sola moltitudine, vol. I, a cura di A. Tabucchi (con la collaborazione di M. J. de Lancastre), Milano, Adelphi, 1979;

Una sola moltitudine, vol. II, a cura di A. Tabucchi (con la collaborazione di M. J. de Lancastre), Milano, Adelphi, 1984;

Il poeta è un fingitore, a cura di G. Tavani, L’Aquila, Japadre, 1988;

Nove poesie di Alvaro de Campos e sette poesie ortonime, a cura di A. Tabucchi, Bologna, Baskerville, 1988;

Faust, a cura di M. J. de Lancastre, Torino, Einaudi, 1989;

Il violinista pazzo, a cura di A. Di Munno, Roma, Lucarini, 1989 (riedizioni: Milano, Mondadori, 1995; Firenze-Antella, Passigli, 2004);

Quartine di gusto popolare, a cura di S. Naglia, Pescara, Tracce, 1992 (riedizione riveduta: Pescara-Roma, L'Unità, 1999 e nuova edizione: Pescara, Tracce, 2011)

Poesie di Álvaro de Campos, a cura di M. J.de Lancastre, Milano, Adelphi, 1993; Epitalamio, a cura di S.Naglia, Pescara, Tracce, 1994;

Alla memoria del Presidente-Re Sidónio Pais, a cura di B. De Cusatis, Roma, Pellicani, 1997 (riedizione: Perugia, Edizioni dell'Urogallo, 2010);

I trentacinque sonetti, a cura di U. Serani, Firenze-Antella, Passigli Editori, 1999;

Poemi di Alberto Caeiro, a cura di P. Raule, Milano, La Vita Felice, 1999; Poesie esoteriche, a cura di F. Zambon, Parma, Guanda Editore, 2000 (riedizione: Milano, TEA, 2002);

Fantasie di interludio. Antologia personale (1914-1935), a cura di F. C. Martins, Firenze-Antella, Passigli Editori, 2002 (riedizione: Nei giorni di luce perfetta, a cura di Paolo Collo, Milano, RCS-Corriere della Sera, 2011);

Poesie di Alberto Caeiro, a cura di F. C. Martins e R. Zenith, FirenzeAntella, Passigli Editori, 2002; 
Messaggio, a cura di F. Cabral Martins (ediz. italiana a cura di P. Collo), Firenze-Antella, Passigli Editori, 2003;

Poesie di Ricardo Reis, a cura di M. Parreira da Silva, Firenze-Antella, Passigli Editori, 2005;

Quartine, a cura di L. Freire, Firenze-Antella, Passigli Editori, 2005;

Sono fluito e altre poesie, a cura di S. Mati, Pistoia, Edizioni Via del Vento, 2005;

Il custode di greggi, a cura di P. Collo, Bagno a Ripoli, Passigli Editori, 2007;

Odi di Ricardo Reis, a cura di L. Corsi, Milano, La Vita Felice, 2007;

Mare del Portogallo e altre poesie, trad. di S. Masin, Pistoia, Edizioni Via del Vento, 2007;

Poemetti erotici, trad. di M. Cavallini, Bagno a Ripoli, Passigli Editori, 2007;

Poesie d'amore di Ricardo Reis, a cura di P. Collo, Bagno a Ripoli, Passigli Editori, 2007;

Poesie, a cura di P. Ceccucci, pref. di J. Saramago, Milano, Rizzoli, 2009; Il mondo che non vedo. Poesie ortonime, a cura di P. Ceccucci, Milano, Rizzoli, 2009;

L'esistenza spirituale. Tutte le poesie ortonime, Milano, Acquaviva, 2009;

Un'affollata solitudine. Poesie eteronime, a cura di P. Ceccucci, Milano, Rizzoli, 2012. 